\title{
Ten-year survival outcomes of patients with potentially resectable gastric cancer: impact of clinicopathologic and treatment-related risk factors
}

\author{
Anna Koumarianou a, Sylvia Krivann, Nikolaos Machairas, , Anastasios Ntavatzikosc, Nikos Pantazis', \\ Dimitrios Schizas ${ }^{b}$, George Martikos ${ }^{b}$, Katerina Kampoli ${ }^{a}$, Evangelos P. Misiakos ${ }^{b}$, Pavlos Patapis ${ }^{b}$, \\ Theodoros Liakakos ${ }^{b}$
}

Attikon University Hospital, National and Kapodistrian University of Athens, Medical School, Athens, Greece

Abstract
Background Despite therapeutic advancements, gastric cancer (GC) remains a leading cause of death worldwide.

Methods This retrospective cohort study statistically analyzed the clinicopathologic characteristics, treatments and outcomes of patients with potentially resectable GC managed at our institution between 2006 and 2010. The STROBE checklist was applied.

Results Preoperative assessment of 164 GC patients (male: female ratio 1.87, median age 65 years) assigned $132(80.5 \%)$ to total $(56 ; 42.4 \%)$ or subtotal $(76 ; 57.6 \%)$ gastrectomy. Resection margins were microscopically tumor-free (R0) in 100 (75.8\%), microscopically infiltrated (R1) in 25 (18.9\%) and macroscopically infiltrated (R2) in 7 (5.3\%) patients. Nodal plane dissection was D0 in $34(25.8 \%)$, D1 in $62(47.0 \%)$ and D2 in $36(27.3 \%)$ patients. Early GC was diagnosed in 19 patients (14.4\%). Fluorouracil-based chemotherapy was administered in $69.7 \%$ and chemoradiation in $18.2 \%$ of patients. The 5 - and 10 -year survival rates of patients with R0 resection were $74 \%$ and $65.4 \%$, respectively. The 2 -year survival rates for R1 and R2 resection were $28.9 \%$ and $0 \%$ respectively. The 5- and 10-year survival rates according to nodal plane dissection were 55.6\% and $41.4 \%$ for D2, and $53.2 \%$ and $49.7 \%$ for D1, respectively. On multivariate analysis, T4, N3 and R1/R2 remained independent negative prognostic factors for overall survival. Microscopic or macroscopic infiltration of surgical margins was the worst adverse prognostic factor for survival.

Conclusion These results are equivalent to those from centers of excellence and indicate the urgent need for improvements in the field, particularly in the development of predictive models to guide personalized therapy.

Keywords Gastric adenocarcinoma, D2 gastrectomy, resection, radiotherapy, multidisciplinary, survival, chemotherapy

Ann Gastroenterol 2019; 32 (1): 1-8
a'Hematology Oncology Unit, Fourth Department of Internal Medicine (Anna Koumarianou, Katerina Kampoli); bThird Department of Surgery (Sylvia Krivan, Nikolaos Machairas, Dimitrios Schizas, George Martikos, Evangelos P. Misiakos, Pavlos Patapis, Theodoros Liakakos); ${ }^{\text {F Fourth }}$ Department of Surgery (Anastasios Ntavatzikos); ${ }^{\mathrm{d} D e p a r t m e n t ~ o f ~}$ Hygiene, Epidemiology and Medical Statistics (Nikos Pantazis), Medical School, National and Kapodistrian University of Athens, Athens, Greece

Conflict of Interest: None

Correspondence to: Anna Koumarianou MD, PhD, Medical Oncology Unit, Fourth Department of Internal Medicine, Attikon University Hospital, Rimini 1, 12462 Athens, Greece, e-mail: akoumari@yahoo.com

Disclosures: Preliminary data from this study were presented in the form of an abstract (e15089) at the Annual Meeting of the American Society of Clinical Oncology, Chicago USA, 2014

Received 24 June 2018; accepted 1 September 2018; published online 3 October 2018

DOI: https://doi.org/10.20524/aog.2018.0320

๑ 2019 Hellenic Society of Gastroenterology

\section{Introduction}

Gastric cancer (GC) remains the fifth most frequent cancer and the third cause of cancer-related deaths worldwide [1,2]. In 2012, an estimated 951,600 new cases of GC were diagnosed, while 723,100 deaths due to GC occurred globally $[1,2]$. Gastric cancer affects twice as many men as women and its incidence presents heterogeneity among different countries: the highest rates are encountered in eastern and western Asia, Latin America, and some former Soviet European countries, and the lowest in Northern America and many parts of Africa [1,2]. In Greece, 1357 deaths from GC were recorded in 2015 (www.statistics.gr). Differences in these rates partly reflect regional discrepancies in several factors, including consumption of fresh products, salt-preserved foods, obesity, alcohol intake, smoking, and incidence of Helicobacter pylori 
(H. pylori) infection, as well as genetic predisposition such as cadherin 1 gene mutation [2-4]. During the recent decades, the incidence of GC has gradually decreased because of primary prevention programs associated with smoking reduction, $H$. pylori infection control and reducing reliance on salt-preserved foods [2]. However, despite the additional introduction of specialized multidisciplinary team (MDT) meetings, the optimization of surgical procedures, advances in systemic chemotherapy, targeted treatments and immunotherapies in the metastatic setting, the overall 5-year survival rates remain low at $20-29 \%$, whereas the median survival of patients with metastatic disease is $9-10$ months [3,5]. An exception is the improved 5-year survival rate of more than 50\% documented in Japan and Korea, due to secondary prevention programs, including endoscopic procedures [5].

Treatment options for patients with GC primarily depend on tumor staging and resectability and derive from a detailed and precise evaluation of imaging and pathology in the context of MDT meetings [6]. Oncologic resection remains the cornerstone of the therapeutic management of GC, but 15-year survival is $21 \%$ for the D1 group and $29 \%$ for the D2 group $(\mathrm{P}=0.34)$ [7]. Aiming to improve these poor results, combination strategies, including chemotherapy, radiotherapy (RT), targeted therapy and immunotherapy, are under intense investigation in the adjuvant, neoadjuvant and metastatic settings. Moreover, following a SWOG-directed Intergroup study, chemoradiation with 5-fluorouracil (5FU) has gained a lot of attention in the United States after curative surgery; nonetheless, concern has been raised about the associated toxicity and benefit of such strategy after optimally performed surgery [8].

The objective of our study was to evaluate the impact of clinical, pathologic and treatment-related risk factors in the survival outcomes of patients with GC from our institution during the period 2006-2010.

\section{Patients and methods}

We conducted a retrospective review of patients assessed for potentially resectable GC at Attikon University General Hospital from 2006-2010. All patients were discussed in our institution's MDT meeting, as per hospital policy, and after thorough clinicopathologic and imaging evaluation, decisions were taken about personalized treatment. As this was a general hospital with two departments of surgery, neither of which was a specialized center for GC, most surgeons had a training background in general surgery. The present study was approved by the institutional review board and written informed consent to the treatment was obtained from all patients, whose data were anonymized and transferred to a dedicated database. The STROBE checklist was applied in this observational study.

\section{Patient data}

Patients were staged using chest/abdominal computed tomography (CT), magnetic resonance imaging and, in selected cases, positron emission tomography-CT prior to surgery to exclude metastatic or locally advanced/unresectable disease. Patients with adenocarcinoma of the esophagogastric junction or stage IV GC (including liver and peritoneal metastasis, extra-regional lymph nodal involvement including the para-aortic and iliac chain) and patients treated with neoadjuvant chemotherapy were excluded from this study. Total gastrectomy was performed for proximal and middle gastric lesions, whereas subtotal gastrectomy was performed for tumors of the distal body, antrum and pylorus, with a minimum surgical resection margin of at least $5 \mathrm{~cm}$. D1 or D2 lymphadenectomy was performed according to the clinical staging: i.e. patients with an anticipated T1N0 or T2N0 tumor underwent a D1 lymphadenectomy, whereas patients with more advanced clinical staging $(>\mathrm{T} 3$ or $\mathrm{N}+$ ) were offered a D2 lymphadenectomy. D1 lymphadenectomy includes dissection of all perigastric lymph nodes plus the left gastric artery lymph nodes; D2 lymphadenectomy includes dissection of perigastric, celiac artery branches and hepatoduodenal ligament lymph nodes; while D0 lymphadenectomy includes anything less than D1 lymphadenectomy. Histopathologic examination of the resected specimens was performed using the GC classification staging system of the American Joint Committee on Cancer, $7^{\text {th }}$ edition. Surgical margins were defined as R0 (no cancer cells identified microscopically at the circular or linear, proximal or distal resection margin); R1 (cancer cells present microscopically at the linear or circular, proximal or distal resection margin); or R2 (tumor tissue seen at the circular or linear, proximal or distal resection margin on gross examination by the naked eye). According to the national clinical recommendations available at the time, adjuvant postoperative chemoradiation was offered to all patients with T3-T4/N0 and T1-T2/N+ tumors, except for patients treated with D2 lymph node dissection, who received only postoperative chemotherapy without RT.

\section{Statistical analysis}

For the purposes of this study, all available patients were included in the analysis. All patients were followed up until death or the final analysis of data. Categorical variables were summarized as absolute and relative (\%) frequencies, whereas median and interquartile range (IQR) were used to summarize the distributions of continuous variables. Survival analysis techniques were used to summarize the time from diagnosis to death and to explore potential associations with demographic and clinical characteristics. More specifically, Kaplan-Meier survival curves and estimated cumulative probabilities of death were used for descriptive purposes, whereas the main analyses were based on univariable and multivariable Cox proportional hazards models and log-rank tests. Survival analyses were performed overall, as well as separately for individuals who underwent surgery and those who did not. No multivariable model was fitted to the subgroup of individuals who did not undergo surgery because of the small size of this group. Poisson modeling was used to explore the association between the number of lymph nodes excised and the year of diagnosis, 
while adjusting for potential confounders. Differences in various clinical characteristics in relation to age (as a categorical variable with three groups) and sex were assessed through chisquare tests. All analyses were performed using Stata 10 (Stata Corp., TX USA).

\section{Results}

\section{Patient characteristics and type of surgery performed}

A total of 164 patients, 59 women and 105 men, were considered for GC resection at our institution between 2006 and 2010. The median age was 65 years (range 56-73). Surgical resection was performed as front-line treatment in 132 patients; 56 (42\%) underwent total gastrectomy and 76 (58\%) subtotal gastrectomy. Although at initial imaging all patients were considered eligible, further evaluation at the MDT meeting indicated metastatic disease in 32 patients (19.5\%) with peritoneal (55\%), lymph nodal (25\%) or liver disease (20\%), diverted from surgery. D0 gastrectomy was performed in 31 (23.5\%), D1 in $62(47 \%)$, D2 in $36(27.3 \%)$, while $3(2.3 \%)$ patients had a palliative operation.

\section{Pathology data}

$\mathrm{T}$ and $\mathrm{N}$ stages for all patients are shown in Table 1. Of the 132 patients operated, $92(70 \%)$ had intestinal and $40(30 \%)$ diffuse adenocarcinoma; 33 (25\%) had proximal and 99 (75\%) distal location; and 52 (39\%) had vascular infiltration and 39 (29\%) perineural invasion. An R0 resection was identified in 100 patients $(75.8 \%)$, whereas $\mathrm{R} 1$ and $\mathrm{R} 2$ resection were recognized in $25(18.9 \%)$ and $7(5.3 \%)$ patients, respectively.

\section{Adjuvant chemotherapy and RT data}

Ninety-two of the 132 patients (70\%) received adjuvant therapy (chemotherapy or chemoradiotherapy) based on the stage and according to the European Society of Medical Oncology's guidelines available at the time. Chemotherapy was offered to all but 1 patient, who did not undergo surgery because of advanced disease and significant comorbidities. There was a range of chemotherapy treatments applied in the adjuvant and metastatic setting, mainly 5FU, platinum and taxane-based as previously reported $[9,10]$. Postoperative RT was offered to $2(6.3 \%)$ patients who did not receive surgical treatment for symptom palliation and to $24(18.2 \%)$ patients who underwent resection and fulfilled the predefined criteria.

\section{Survival outcomes}

At the time of analysis, the median follow-up time was 114.8 months (range 4.4-195) and the median survival time
Table 1 Description of clinicopathologic characteristics of patients according to whether surgery was performed or not

\begin{tabular}{|c|c|c|c|}
\hline \multirow{3}{*}{$\begin{array}{l}\text { Clinicopathologic } \\
\text { characteristics }\end{array}$} & \multicolumn{3}{|c|}{ Surgery performed } \\
\hline & No N (\%) & Yes N (\%) & Total N (\%) \\
\hline & $32(100.0)$ & $132(100.0)$ & $164(100.0)$ \\
\hline \multicolumn{4}{|l|}{ Sex } \\
\hline Female & $13(40.6)$ & $46(34.8)$ & $59(36.0)$ \\
\hline Male & $19(59.4)$ & $86(65.2)$ & $105(64.0)$ \\
\hline \multicolumn{4}{|l|}{ Age } \\
\hline$<50$ & $3(9.4)$ & $17(12.9)$ & $20(12.2)$ \\
\hline $50+$ & $29(90.6)$ & $115(87.1)$ & $144(87.8)$ \\
\hline \multicolumn{4}{|l|}{ Type of surgery } \\
\hline Total gastrectomy & $0(0.0)$ & $56(42.4)$ & $56(34.1)$ \\
\hline Subtotal gastrectomy & $0(0.0)$ & $76(57.6)$ & $76(46.3)$ \\
\hline Not performed & $32(100.0)$ & $0(0.0)$ & $32(19.5)$ \\
\hline \multicolumn{4}{|l|}{ T stage } \\
\hline $\mathrm{T} 1$ & $0(0.0)$ & $19(14.4)$ & $19(11.6)$ \\
\hline $\mathrm{T} 2$ & $0(0.0)$ & $19(14.4)$ & 19 (11.6) \\
\hline $\mathrm{T} 3$ & $0(0.0)$ & $47(35.6)$ & $47(28.7)$ \\
\hline $\mathrm{T} 4$ & $32(100.0)$ & $47(35.6)$ & $79(48.2)$ \\
\hline \multicolumn{4}{|l|}{$\mathrm{N}$ stage } \\
\hline No & $0(0.0)$ & $40(30.3)$ & $40(24.4)$ \\
\hline N1 & $0(0.0)$ & $16(12.1)$ & $16(9.8)$ \\
\hline N2 & $0(0.0)$ & $23(17.4)$ & $23(14.0)$ \\
\hline N3a & $0(0.0)$ & $27(20.5)$ & $27(16.5)$ \\
\hline $\mathrm{N} 3 \mathrm{~b}$ & $0(0.0)$ & $26(19.7)$ & $26(15.9)$ \\
\hline $\mathrm{Nx}$ & $32(100.0)$ & $0(0.0)$ & $32(19.5)$ \\
\hline \multicolumn{4}{|l|}{ Perineural infiltration } \\
\hline No & $0(0.0)$ & $93(70.5)$ & $93(56.7)$ \\
\hline Yes & $0(0.0)$ & $39(29.5)$ & $39(23.8)$ \\
\hline NA & $32(100.0)$ & $0(0.0)$ & $32(19.5)$ \\
\hline \multicolumn{4}{|l|}{ Vascular invasion } \\
\hline No & $0(0.0)$ & $80(60.6)$ & $80(48.8)$ \\
\hline Yes & $0(0.0)$ & $52(39.4)$ & $52(31.7)$ \\
\hline NA & $32(100.0)$ & $0(0.0)$ & $32(19.5)$ \\
\hline \multicolumn{4}{|l|}{ Resection margins } \\
\hline R0 & $0(0.0)$ & $100(75.8)$ & $100(61.0)$ \\
\hline $\mathrm{R} 1$ & $0(0.0)$ & $25(18.9)$ & $25(15.2)$ \\
\hline $\mathrm{R} 2$ & $0(0.0)$ & $7(5.3)$ & $7(4.3)$ \\
\hline Surgery not done & $32(100.0)$ & $0(0.0)$ & $32(19.5)$ \\
\hline \multicolumn{4}{|l|}{ Plane of nodal dissection } \\
\hline D0 & $0(0.0)$ & $31(23.5)$ & $31(18.9)$ \\
\hline D1 & $0(0.0)$ & $62(47.0)$ & $62(37.8)$ \\
\hline
\end{tabular}

(Contd...) 
Table 1 (Continued)

\begin{tabular}{lccc}
\hline \multirow{2}{*}{$\begin{array}{l}\text { Clinicopathologic } \\
\text { characteristics }\end{array}$} & \multicolumn{3}{c}{ Surgery performed } \\
\cline { 2 - 4 } & No N (\%) & Yes N (\%) & Total N (\%) \\
\cline { 2 - 4 } & $32(100.0)$ & $132(100.0)$ & $164(100.0)$ \\
\hline D2 & $0(0.0)$ & $36(27.3)$ & $36(22.0)$ \\
Not done & $32(100.0)$ & $3(2.3)$ & $35(21.3)$ \\
Chemotherapy & & & \\
No & $1(3.1)$ & $40(30.3)$ & $41(25.0)$ \\
Yes & $31(96.9)$ & $92(69.7)$ & $123(75.0)$ \\
Radiotherapy & & & \\
No & $30(93.8)$ & $108(81.8)$ & $138(84.1)$ \\
Yes & $2(6.3)$ & $24(18.2)$ & $26(15.9)$ \\
\hline
\end{tabular}

$\mathrm{N}$, number of patients

was 47.6 months (95\% confidence interval [CI] 27.5-70.5). For patients who underwent surgery, the median follow-up and survival times were 114.8 (range 5.1-195.0) and 100.3 (95\%CI 54.0-195.0+) months, respectively. In contrast, the median follow-up and survival times in patients who did not undergo surgery were 27 (range 4.4-37.0) and 13 (95\%CI 10.0-17.1) months, respectively. The survival time since diagnosis and by surgical margins, plane of nodal dissection and TNM, are shown in Table 2. The Kaplan-Meier survival curve in relation to surgical margin involvement is shown in Fig. 1A. The mean (95\% CI) survival rates at 5 years post diagnosis for T3 and T4 were 57.4 (42.1-70.1) and 25.5 (14.2-38.5), whereas for N2, $\mathrm{N} 3 \mathrm{a}$ and N3b they were 56.5 (34.3-73.8), 37.0 (19.6-54.6) and 15.4 (4.8-31.5), respectively. The mean (95\%CI) survival at 5 years post diagnosis was $85.0(69.6,93.0), 81.3$ (52.5-93.5), 56.5 (34.3-73.8), 37.0 (19.6-54.6) and 15.4 (4.8-31.5) according to the presence of $0,1-2,3-6,7-15$ and $>16$ infiltrated lymph nodes (Fig. 1B).

The mean $(95 \% \mathrm{CI})$ survival at 5 -years post diagnosis in relation to the absence or presence of vascular infiltration was 61.3 (49.7-70.9) and 48.1 (34.1-60.8), whereas for absence or presence of perineural invasion it was 61.3 (50.6-70.3) and 43.6 (27.9-58.3), respectively. The Kaplan-Meier survival curve according to TNM stage is shown in Fig. 1C.

The association of the surgical margin status with $\mathrm{N}$ stage revealed that there was a statistically significant difference $(\mathrm{P}<0.001)$ in the mean $(95 \% \mathrm{CI})$ survival at 2 years post diagnosis between patients in the subgroup including R1/ $\mathrm{R} 2 \& \mathrm{~N} 0 / \mathrm{N} 1 / \mathrm{N} 2[11.1(0.6-38.8)]$ and those with R0 \& N3 [83.3 (64.5-92.7)] disease. The respective Kaplan-Meier survival curves are shown in Fig. 1D.

On univariate Cox regression analysis of patients treated with surgery, T3 and T4 tumors, N2/N3 tumors, perineural infiltration, R1 and R2 resection, chemotherapy and RT were associated adversely with survival to a degree that reached statistical significance (Table 3). Radiotherapy was associated with increased risk of death but this finding was not confirmed in the multivariate analysis. On multivariate Cox regression analysis, patients treated with surgery, T3 and T4 tumors, N3 stage, R1 and R2 resection reached statistical significance and remained independent negative prognostic factors for overall survival (Table 4).

\section{Discussion}

Data on the long-term survival of patients with GC initially considered to be resectable are important and provide a reference point for the evaluation of advanced therapeutic strategies. Our study provides findings concerning the epidemiologic, clinicopathologic and 10-year survival data of patients with potentially resectable GC. This is the first realworld data reporting on patient outcomes from Greece.

Our patients had a male-to-female ratio of 1.87 . This is in agreement with a retrospective analysis of 534 patients with stage III GC from Taiwan [11]. The patients' median age and location rates (proximal vs. distal) were similar to those in previously reported prospective and retrospective studies of patients with resectable GC $[12,13]$.

Adenocarcinoma subtypes were assessed according to the Lauren classification and the intestinal-to-diffuse rate was found to be similar to that in a recent study reporting on 534 patients from Taiwan [11]. Our study was not designed to identify survival benefit in relation to chemotherapy, but a recent report on a GC registry indicated that tumor classification according to Lauren predicted not only survival, but also response to chemotherapy, as docetaxel was associated with improved progression-free survival and overall survival in patients with intestinal adenocarcinoma [14]. The optimal combination and sequence of chemotherapy in the treatment algorithm is under intense investigation, but perioperative chemotherapy is currently gaining ground, particularly for T3/T4a and/or regional lymph node positive tumors [15]. In this context, preoperative FLOT, a docetaxel-based triplet chemotherapy, was compared to ECF/ECX anthracyclinebased triplet chemotherapy and was found to be superior, as it was associated with significantly higher proportions of pathologic complete regression [15].

In the univariate analysis of our study, vascular and perineural invasion were found to be associated with an increased risk of death. This effect was not maintained in the multivariate analysis, most probably because of the small numbers of patients, but a previous study including 734 patients who underwent surgery indicated that both vascular and perineural invasion were independent prognostic factors for disease-free and overall survival [16].

Another important pathological aspect influencing the clinical outcome of GC patients was found to be microscopic and macroscopic tumoral infiltration of surgical margins. In the univariate analysis, both $\mathrm{R} 1$ and $\mathrm{R} 2$ resection were associated with a statistically significantly higher risk of death, an effect also maintained in the multivariate analysis as one of the most important predictors of relapse and death. It is well established that the effectiveness of surgery depends on the adequacy of the surgical procedures and on a cancer-free surgical margin 
Table 2 Long-term survival outcomes of patients according to TNM stage, plane of nodal dissection and status of surgical resection margins

\begin{tabular}{|c|c|c|c|}
\hline \multirow[t]{2}{*}{ Clinicopathologic characteristics } & \multicolumn{3}{|c|}{$\%$ of patients alive ( $95 \%$ confidence interval) } \\
\hline & 36 months & 60 months & 120 months \\
\hline \multicolumn{4}{|l|}{ pTNM stage } \\
\hline IIA & $93.8(63.2,99.1)$ & $93.8(63.2,99.1)$ & $93.8(63.2,99.1)$ \\
\hline IIB & $83.3(48.2,95.6)$ & $66.7(33.7,86.0)$ & $66.7(33.7,86.0)$ \\
\hline IIIA & $72.2(45.6,87.4)$ & $44.4(21.6,65.1)$ & $38.1(16.6,59.5)$ \\
\hline IIIB & $57.1(39.3,71.5)$ & $34.3(19.3,49.8)$ & $17.6(6.6,32.9)$ \\
\hline IIIC & $20.8(7.6,38.5)$ & $16.7(5.2,33.7)$ & $12.5(3.1,28.7)$ \\
\hline \multicolumn{4}{|l|}{ Nodal dissection } \\
\hline D0 & $71.0(51.6,83.7)$ & $64.5(45.2,78.5)$ & $60.2(40.4,75.3)$ \\
\hline D1 & $67.7(54.6,77.8)$ & $53.2(40.1,64.7)$ & $49.7(36.7,61.4)$ \\
\hline $\mathrm{D} 2$ & $69.4(51.7,81.8$ & $55.6(38.1,69.9)$ & $41.4(24.3,57.7)$ \\
\hline Not done & $33.3(0.9,77.4)$ & $0(0,0)$ & $0(0,0)$ \\
\hline \multicolumn{4}{|l|}{ Resection margins } \\
\hline \multirow[t]{2}{*}{ R0 } & $90.0(82.2,94.5)$ & $74.0(64.2,81.5)$ & $65.4(54.8,74.2)$ \\
\hline & 12 months & 18 months & 24 months \\
\hline $\mathrm{R} 1$ & $68.0(46.1,82.5)$ & $32.0(15.2,50.2)$ & $12.0(3.0,27.7)$ \\
\hline R2 & $85.7(33.4,97.9)$ & $42.9(9.8,73.4)$ & $0.0(0.0,0.0)$ \\
\hline
\end{tabular}

Stage IA and IB are not shown as there were no deaths, HR was 0 and upper limit of confidence interval could not be estimated
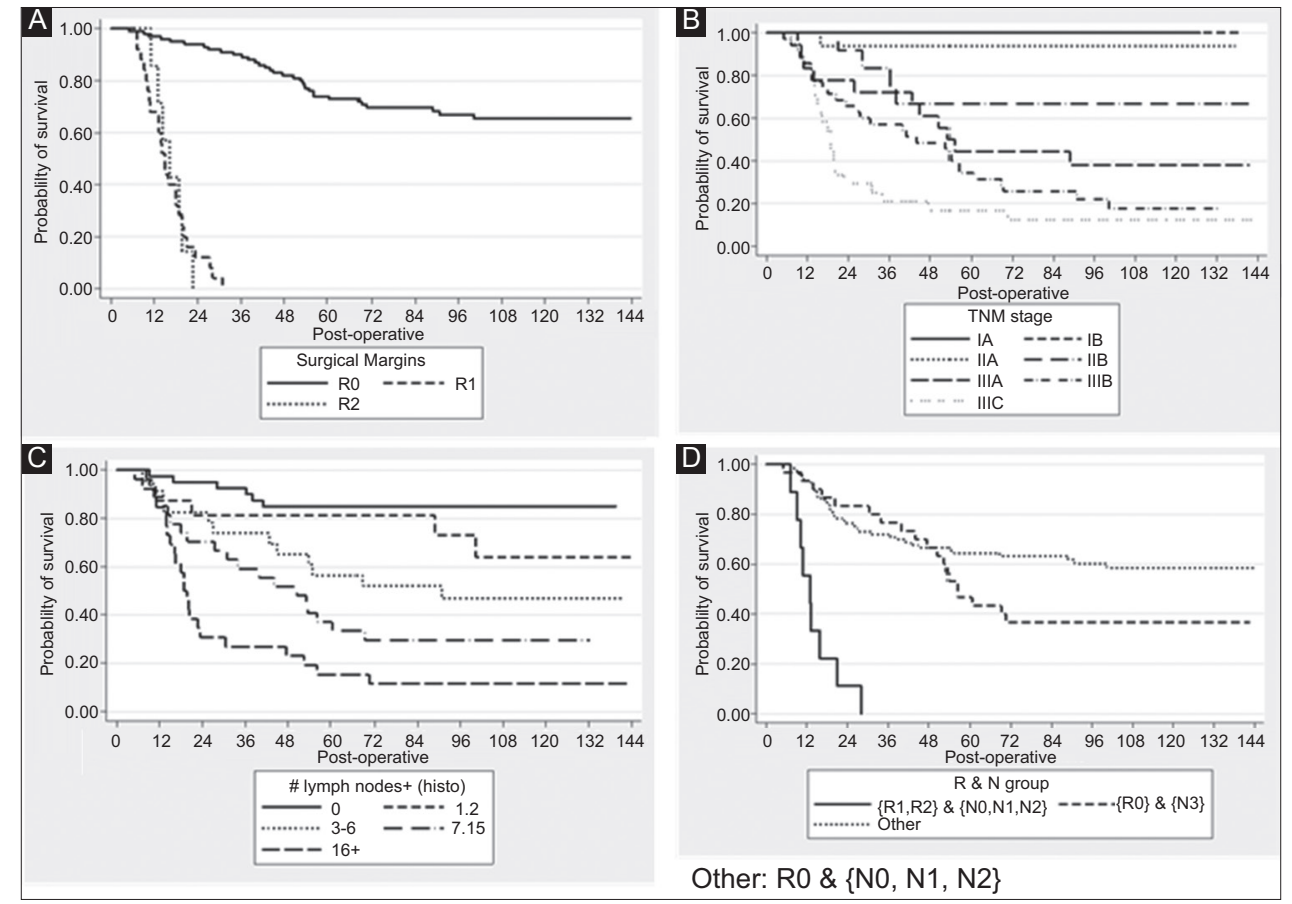

Figure 1 Kaplan-Meier survival curves according to: (A) infiltration of surgical margins; (B) number of involved lymph nodes; (C) TNM stages (7th AJCC edition); (D) resection margin (R0, R1, R2) and nodal (N0, N1, N2, N3) infiltration

specimen (R0), as patients with $\mathrm{R} 1$ or $\mathrm{R} 2$ resection have a mean survival of 8.7 months [17]. More importantly, the analysis of patients according to $\mathrm{N}$ stage and margin infiltration indicated that patients with margin infiltration had far worse survival when compared to patients with N2 disease. Similarly to our study, achieving R0 resection was also found to be significant in a recent study including 82 patients with gastric cardia cancer and R1/R2 resection was found to be associated with 
Table 3 Univariate cox regression analysis of prognostic factors in patients treated with surgery

\begin{tabular}{|c|c|c|c|}
\hline Factor & $\begin{array}{l}\text { Hazard } \\
\text { ratio }\end{array}$ & $\begin{array}{l}95 \% \text { confidence } \\
\text { interval }\end{array}$ & $\mathrm{P}$-value \\
\hline
\end{tabular}

\begin{tabular}{lccc}
\hline Sex & & & \\
Female & 0.98 & $(0.58,1.63)$ & 0.925 \\
Male $^{*}$ & 1 & & \\
Age & & & \\
$<50^{*}$ & 1 & & \\
$50+$ & 1.05 & $(0.52,2.12)$ & 0.901
\end{tabular}

Type of surgery

$\begin{array}{lccc}\text { Total gastrectomy } & 1 & & \\ \text { Subtotal gastrectomy } & 0.63 & (0.39,1.03) & 0.064\end{array}$

$\begin{array}{cccc}\text { T stage } & & & \\ \mathrm{T}^{*} & 1 & & \\ \mathrm{~T} 3 & 3.77 & (1.13,12.61) & 0.031 \\ \mathrm{~T} 4 & 10.39 & (3.20,33.76) & <0.001\end{array}$

$\mathrm{N}$ stage
$\mathrm{N} 0^{*}$

$\begin{array}{lccc}\text { N1 } & 2.24 & (0.68,7.33) & 0.184 \\ \text { N2 } & 4.26 & (1.60,11.35) & 0.004 \\ \text { N3a } & 6.85 & (2.73,17.20) & <0.001 \\ \text { N3b } & 13.24 & (5.33,32.89) & <0.001\end{array}$

$\begin{array}{lccc}\begin{array}{l}\text { Perineural infiltration } \\ \text { No* }\end{array} & 1 & & \\ \text { Yes } & 1.76 & (1.07,2.91) & 0.026 \\ \text { Vascular invasion } & & & \\ \text { No* } & 1 & & \\ \text { Yes } & 1.59 & (0.98,2.59) & 0.062 \\ \text { Resection margins } & & & \\ \text { R0* } & 1 & & \\ \text { R1 } & 35.01 & (15.37,79.75) & <0.001 \\ \text { R2 } & 35.48 & (12.11,103.95) & <0.001\end{array}$

Plane of nodal dissection

\begin{tabular}{lccc} 
D0 & 1 & & \\
D1 & 1.36 & $(0.70,2.66)$ & 0.363 \\
D2 & 1.49 & $(0.73,3.05)$ & 0.278 \\
Not done & 2.61 & $(0.58,11.69)$ & 0.210 \\
Chemotherapy & & & \\
No & 1 & & \\
Yes & 21.17 & $(5.17,86.69)$ & $<0.001$ \\
Radiotherapy & & & \\
No* & 1 & & \\
Yes & 1.84 & $(1.04,3.23)$ & 0.035 \\
\hline * Reference group & & & \\
\hline
\end{tabular}

T1 stage is not shown as there were no deaths, HR was 0 and upper limit of confidence interval could not be estimated
Table 4 Multivariate cox regression analysis of prognostic factors in patients treated with surgery

\begin{tabular}{|c|c|c|c|}
\hline Factor & $\begin{array}{l}\text { Hazard } \\
\text { ratio }\end{array}$ & $\begin{array}{l}95 \% \text { confidence } \\
\text { interval }\end{array}$ & P-value \\
\hline \multicolumn{4}{|l|}{ Age } \\
\hline per 10 years & 0.98 & $(0.79,1.22)$ & 0.854 \\
\hline \multicolumn{4}{|l|}{ Sex } \\
\hline Female & 0.92 & $(0.50,1.68)$ & 0.781 \\
\hline Male $^{*}$ & 1 & & \\
\hline \multicolumn{4}{|l|}{ T stage } \\
\hline $\mathrm{T} 2^{*}$ & 1 & & \\
\hline T3 & 1.91 & $(0.53,6.85)$ & 0.322 \\
\hline $\mathrm{T} 4$ & 3.32 & $(0.93,11.86)$ & 0.064 \\
\hline \multicolumn{4}{|l|}{$\mathrm{N}$ stage } \\
\hline $\mathrm{N}^{*}$ & 1 & & \\
\hline $\mathrm{N} 1$ & 1.80 & $(0.51,6.32)$ & 0.357 \\
\hline $\mathrm{N} 2$ & 2.51 & $(0.83,7.53)$ & 0.102 \\
\hline N3 & 2.95 & $(1.11,7.85)$ & 0.030 \\
\hline \multicolumn{4}{|c|}{ Resection margins } \\
\hline $\mathrm{R} 0^{*}$ & 1 & & \\
\hline R1 & 20.98 & $(8.37,52.60)$ & $<0.001$ \\
\hline R2 & 15.39 & $(4.57,51.82)$ & $<0.001$ \\
\hline \multicolumn{4}{|c|}{ Plane of nodal dissection } \\
\hline D0 & 1 & & \\
\hline D1 & 0.77 & $(0.35,1.73)$ & 0.532 \\
\hline D2 & 0.82 & $(0.33,2.01)$ & 0.660 \\
\hline Not done & 1.36 & $(0.25,7.45)$ & 0.726 \\
\hline
\end{tabular}

* Reference group

T1 stage is not shown as there were no deaths, HR was 0 and upper limit of confidence interval could not be estimated

a 5-year survival rate of $13 \%$ [18]. Achieving an R0 resection is of the utmost importance, but is not always feasible. Twelve studies included in a systematic review, reporting on a total of 15,008 patients, indicated that intraoperative frozen sections should be performed to achieve a negative margin with intraoperative re-excision and that surgical re-excision of an R1 resection should be considered for patients with fewer than three disease-positive nodes, because survival is more likely to be governed by positive margins than by nodal status [19]. However, although management of the surgical margin seems to be of paramount importance in the early stage, at a later stage of the disease, when additional adverse pathologic characteristics (such as N3 disease) are present and ultimately determine patients' outcomes, the decision to extend a resection to achieve a cancer-free resection margin should be considered carefully and personalized [20].

The univariate analysis of this study found signs of a possible negative association of RT with survival. This is somewhat misleading, as most patients receiving RT had positive lymph nodes or infiltrated surgical margins and thus a more 
unfavorable disease prognosis. Despite several clinical studies, the role of RT in resected GC remains controversial $[8,9,21]$. A previous study from Europe that randomized patients with histologically proven, radically resected GC, stage $\geq \mathrm{T} 3$ and $\geq \mathrm{N} 1$, to receive 6 cycles of docetaxel with cisplatin, both at $75 \mathrm{mg} / \mathrm{m}^{2}$ every 3 weeks ( $\operatorname{arm~A}$ ), or the same treatment with RT (45 Gy; arm B) showed no differences in overall and disease-free survival between the two arms. [9]. Similarly no survival benefit was found in the ARTIST trial from Korea, which randomized patients with resected GC to receive 6 cycles of capecitabine $1000 \mathrm{mg} / \mathrm{m}^{2}$ twice a day on days 1 to 14 and cisplatin $60 \mathrm{mg} / \mathrm{m}^{2}$ on day 1 every 3 weeks ( $\operatorname{arm~A}$ ), or two cycles of the same chemotherapy followed by RT 45 Gy concurrently with capecitabine $825 \mathrm{mg} / \mathrm{m}^{2}$ twice a day, followed by two additional cycles of chemotherapy (arm B) [21]. The USA 0116 SWOG-directed intergroup trial randomized patients to surgery alone (arm A) or postoperative chemoradiotherapy, including bolus $5 \mathrm{FU} 425 \mathrm{mg} / \mathrm{m}^{2} / \mathrm{d}$ and leucovorin $20 \mathrm{mg} / \mathrm{m}^{2} / \mathrm{d}$ on days 1 through 5 before, during, and after RT to a total of $45 \mathrm{~Gy}$ (1.8 Gy/d $5 \mathrm{~d} /$ wk for 5 weeks), targeting common locoregional failure sites such as the tumor bed, regional nodes, and anastomoses [8]. Two major differences between the later study and the previous ones were the administration of $5 \mathrm{FU}$ based chemotherapy and the inclusion of $85 \%$ of patients with N1-N3 disease (only 10\% of patients had D2 dissection). An important randomized study, which aims to compare TS-1 (4060 mg b.i.d.; 4 weeks - 2 weeks off; 8 cycles), TS-1 (40-60 mg b.i.d.; 2 weeks - 1 week off $)+$ oxaliplatin $\left(130 \mathrm{mg} / \mathrm{m}^{2} \mathrm{q} 3\right.$ weeks $)$ both for 8 cycles, with or without RT (45 Gy in 5 weeks) in D2 resected GC, is currently underway to shed light on the need for RT in optimally resected patients (ClinicalTrials.gov Identifier: NCT01761461).

Our study has some limitations, as it included only a small number of patients in each subgroup. In our analysis, we identified 34 patients with stage I-II GC who were not treated with nodal dissection. This D0 subgroup had a 10-year survival benefit of $60 \%$, significantly greater than the D1 and D2 survival values achieved. This result is misleading and should be interpreted with caution, as the subgroup of patients with stage I-II and lymph node negative disease had a better natural history and survival [22]. Based on the patient's performance status and clinical evidence, D1 or D2 resection represents the recommended surgical procedure [23]. Likewise, as our analysis included patients with T1-T2 and node-negative disease who did not receive chemotherapy or RT, overall patient survival was better among those not treated with chemotherapy or RT than in those who were treated, a misleading effect due to the better prognosis of this subgroup of patients.

Notwithstanding the evolution of endoscopic and surgical techniques, improved molecular targeted therapies and chemoradiation, GC remains a leading cause of death worldwide. Multimodal planning of GC management, including surgeons, medical and radiation oncologists, radiologists and pathologists, is imperative and of cardinal importance. Despite improvements in radical resection with tumor-free margins and extended lymph node dissection, patients' survival prospects remain poor. The prognosis of GC is influenced by a variety of additional tumor-, patient- and therapy-related factors, such as involvement of the surgical margins, lymphovascular invasion, number of lymph nodes involved and molecular signature, regardless of the type of lymphadenectomy [3]. Molecular studies have yielded a vast quantity of new information for the potential exploitation of novel molecules targeting the different GC subtypes [24,25]. Three randomized studies (the Dutch, the FLOT4/AIO and the CRITICS studies) have provided proof of the superiority of perioperative administration of FLOT regimen and gastrectomy with D2 lymphadenectomy over adjuvant postoperative chemoradiotherapy $[7,15,26]$. These findings offer new therapeutic options expected to supersede the 0116 SWOG trial protocol, the previous standard of care, including adjuvant chemoradiotherapy [8]. Finally, as detailed immune profiling of GC is yielding promising results, early studies with immune checkpoint inhibitors in advanced disease suggest that GC may be amenable to immune modulation $[27,28]$.

Our study provides additional information about the survival of patients with GC, treated in a real-world environment, and confirms the importance of TNM staging for survival, as more advanced tumors (T4, N2 and N3) were

\section{Summary Box}

\section{What is already known:}

- Gastric cancer (GC) remains the fifth most frequent cancer and the third cause of cancer-related deaths worldwide

- The heterogeneity in the incidence of GC among countries is due to differences in fresh product consumption, salt-preserved foods, obesity, alcohol intake, smoking, incidence of Helicobacter pylori infection and genetic predisposition, such as cadherin 1 gene mutation

- Since 1991, the cornerstone of treatment in GC has been gastrectomy with lymphadenectomy, followed by adjuvant chemoradiotherapy, according to the USA 0116 SWOG-directed intergroup trial

- The identification of GC molecular subtypes defines sets of patients (Epstein-Barr Virus positive, microsatellite unstable, genomically stable and chromosomally unstable) for targeted therapy trials

\section{What the new findings are:}

- Gastrectomy with D1 or D2 lymphadenectomy, significantly improves the survival of patients with GC and is the optimal surgery for patients with resectable GC

- Microscopic and macroscopic infiltration of surgical margins are the worst negative prognostic factors for survival. Achieving R0 surgical resection is of the utmost importance 
found to be associated with worse outcomes on multivariate analysis. Inclusion criteria and analyses of future clinical trials must reevaluate the role of surgery, RT and chemotherapy in relation to tumor location, Lauren histology and the newly developed molecular profiling, as reported in the seminal Cancer Genome Atlas Study.

\section{References}

1. Ferlay J, Soerjomataram I, Dikshit R, et al. Cancer incidence and mortality worldwide: sources, methods and major patterns in GLOBOCAN 2012. Int J Cancer 2015;136:E359-E386.

2. Torre LA, Siegel RL, Ward EM, Jemal A. Global cancer incidence and mortality rates and trends-an update. Cancer Epidemiol Biomarkers Prev 2016;25:16-27.

3. Ajani JA, Lee J, Sano T, Janjigian YY, Fan D, Song S. Gastric adenocarcinoma. Nat Rev Dis Primers 2017;3:17036.

4. Oliveira C, Pinheiro H, Figueiredo J, Seruca R, Carneiro F. Familial gastric cancer: genetic susceptibility, pathology, and implications for management. Lancet Oncol 2015;16:e60-e70.

5. Allemani C, Matsuda T, Di Carlo V, et al; CONCORD Working Group. Global surveillance of trends in cancer survival 2000-14 (CONCORD-3): analysis of individual records for 37513025 patients diagnosed with one of 18 cancers from 322 population-based registries in 71 countries. Lancet 2018;391:1023-1075.

6. Davies AR, Deans DA, Penman I, et al. The multidisciplinary team meeting improves staging accuracy and treatment selection for gastro-esophageal cancer. Dis Esophagus 2006;19:496-503.

7. Songun I, Putter H, Kranenbarg EM, Sasako M, van de Velde CJ. Surgical treatment of gastric cancer: 15-year follow-up results of the randomised nationwide Dutch D1D2 trial. Lancet Oncol 2010;11:439-449.

8. Smalley SR, Benedetti JK, Haller DG, et al. Updated analysis of SWOG-directed intergroup study 0116: a phase III trial of adjuvant radiochemotherapy versus observation after curative gastric cancer resection. J Clin Oncol 2012;30:2327-2333.

9. Bamias A, Karina M, Papakostas P, et al. A randomized phase III study of adjuvant platinum/docetaxel chemotherapy with or without radiation therapy in patients with gastric cancer. Cancer Chemother Pharmacol 2010;65:1009-1021.

10. Papakostas P, Tsavdaridis D, Kosmidis P, et al. Combination docetaxel (Taxotere), fluorouracil, and leucovorin (TFL), as first-line chemotherapy in advanced gastric cancer: a Hellenic Cooperative Oncology Group phase II study. Gastric Cancer 2006;9:26-31.

11. Chang SC, Liu KH, Hung CY, et al. Adjuvant chemotherapy improves survival in stage III gastric cancer after D2 surgery. J Cancer 2018;9:81-91.

12. Cunningham D, Allum WH, Stenning SP, et al. Perioperative chemotherapy versus surgery alone for resectable gastroesophageal cancer. N Engl J Med 2006;355:11-20.

13. Kanhere H, Goel R, Finlay B, Trochsler M, Maddern G. Radical gastrectomy: still the cornerstone of curative treatment for gastric cancer in the perioperative chemotherapy era-a single institute experience over a decade. Int J Surg Oncol 2018;2018:9371492.

14. Jiménez Fonseca P, Carmona-Bayonas A, Hernández R, et al. Lauren subtypes of advanced gastric cancer influence survival and response to chemotherapy: real-world data from the AGAMENON National Cancer Registry. Br J Cancer 2017;117:775-782.

15. Al-Batran SE, Hofheinz RD, Pauligk C, et al. Histopathological regression after neoadjuvant docetaxel, oxaliplatin, fluorouracil, and leucovorin versus epirubicin, cisplatin, and fluorouracil or capecitabine in patients with resectable gastric or gastrooesophageal junction adenocarcinoma (FLOT4-AIO): results from the phase 2 part of a multicentre, open-label, randomised phase $2 / 3$ trial. Lancet Oncol 2016;17:1697-1708.

16. Scartozzi M, Galizia E, Verdecchia L, et al. Lymphatic, blood vessel and perineural invasion identifies early-stage high-risk radically resected gastric cancer patients. Br J Cancer 2006;95:445-449.

17. Yoo CH, Noh SH, Shin DW, Choi SH, Min JS. Recurrence following curative resection for gastric carcinoma. Br J Surg 2000;87:236-242.

18. Ito H, Clancy TE, Osteen RT, et al. Adenocarcinoma of the gastric cardia: what is the optimal surgical approach? J Am Coll Surg 2004;199:880-886.

19. Aurello P, Magistri P, Nigri G, et al. Surgical management of microscopic positive resection margin after gastrectomy for gastric cancer: a systematic review of gastric R1 management. Anticancer Res 2014;34:6283-6288.

20. Postlewait LM, Maithel SK. The importance of surgical margins in gastric cancer. J Surg Oncol 2016;113:277-282.

21. Park SH, Sohn TS, Lee J, et al. Phase III trial to compare adjuvant chemotherapy with capecitabine and cisplatin versus concurrent chemoradiotherapy in gastric cancer: final report of the adjuvant chemoradiotherapy in stomach tumors trial, including survival and subset analyses. J Clin Oncol 2015;33:3130-3136.

22. Ahn HS, Lee HJ, Hahn S, et al. Evaluation of the seventh American Joint Committee on Cancer/International Union Against Cancer Classification of gastric adenocarcinoma in comparison with the sixth classification. Cancer 2010;116:5592-5598.

23. De Manzoni G, Marrelli D, Baiocchi GL, et al. The Italian Research Group for Gastric Cancer (GIRCG) guidelines for gastric cancer staging and treatment: 2015. Gastric Cancer 2017;20:20-30.

24. Cancer Genome Atlas Research Network. Comprehensive molecular characterization of gastric adenocarcinoma. Nature 2014;513:202-209.

25. Cristescu R, Lee J, Nebozhyn M, et al. Molecular analysis of gastric cancer identifies subtypes associated with distinct clinical outcomes. Nat Med 2015;21:449-456.

26. Cats A, Jansen EPM, van Grieken NCT, et al; CRITICS investigators. Chemotherapy versus chemoradiotherapy after surgery and preoperative chemotherapy for resectable gastric cancer (CRITICS): an international, open-label, randomised phase 3 trial. Lancet Oncol 2018;19:616-628.

27. Kang YK, Boku N, Satoh T, et al. Nivolumab in patients with advanced gastric or gastro-oesophageal junction cancer refractory to, or intolerant of, at least two previous chemotherapy regimens (ONO-4538-12, ATTRACTION-2): a randomised, double-blind, placebo-controlled, phase 3 trial. Lancet 2017;390:2461-2471.

28. Le DT, Durham JN, Smith KN, et al. Mismatch repair deficiency predicts response of solid tumors to PD-1 blockade. Science 2017;357:409-413. 